The Astrophysical Journal, 671:2017-2027, 2007 December 20

(C) 2007. The American Astronomical Society. All rights reserved. Printed in U.S.A.

\title{
MID-INFRARED INTERFEROMETRY OF DUST AROUND MASSIVE EVOLVED STARS
}

\author{
Jayadev Rajagopal, ${ }^{1}$ Jean-Luc Menut, ${ }^{2}$ D. Wallace, ${ }^{3}$ W. C. Danchi, ${ }^{3}$ O. Chesneau, ${ }^{4}$ \\ B. Lopez, ${ }^{2}$ J. D. Monnier, ${ }^{5}$ M. Ireland, ${ }^{6}$ and P. G. Tuthill ${ }^{7}$ \\ Received 2007 April 22; accepted 2007 August 15
}

\begin{abstract}
We report long-baseline interferometric measurements of circumstellar dust around massive evolved stars with the MIDI instrument on the Very Large Telescope Interferometer and provide spectrally dispersed visibilities in the 8-13 $\mu \mathrm{m}$ wavelength band. We also present diffraction-limited observations at $10.7 \mu \mathrm{m}$ on the Keck Telescope with baselines up to $8.7 \mathrm{~m}$, which explore larger scale structure. We have resolved the dust shells around the late-type WC stars WR 106 and WR 95 and the enigmatic NaSt 1 (formerly WR 122), suspected to have recently evolved from a luminous blue variable (LBV) stage. For AG Car, the prototypical LBV in our sample, we marginally resolve structure close to the star, distinct from the well-studied detached nebula. The dust shells around the two WC stars show fairly constant size in the 8-13 $\mu \mathrm{m}$ MIDI band, with Gaussian half-widths of $\sim 25$ to 40 mas, and the Keck observations reveal an additional extended structure around WR 106. The visibility profiles for NaSt 1 obtained from two MIDI baselines indicate a compact source embedded in an extended structure. The compact dust we detect around NaSt 1 and AG Car favors recent or ongoing dust formation. Using the measured visibilities, we build spherically symmetric radiative transfer models of the WC dust shells, which enable detailed comparison with existing SED-based models. Our results indicate that the inner radii of the shells are within a few tens of AU from the stars. In addition, our models favor grain size distributions with large $(\sim 1 \mu \mathrm{m})$ dust grains. This proximity of the inner dust to the hot central star emphasizes the difficulty faced by current theories in forming dust in the hostile environment around WR stars. Although we detect no direct evidence for binarity for these objects, dust production in a colliding-wind interface in a binary system is a feasible mechanism in WR systems under these conditions.
\end{abstract}

Subject headings: stars: individual (AG Car, NaSt 1, WR 95, WR 106) — techniques: interferometric

\section{INTRODUCTION}

The most massive stars $\left(\gtrsim 40 M_{\odot}\right)$ will likely pass through a luminous blue variable (LBV) and Wolf-Rayet (WR) phase before ending their lives in a supernova explosion. LBVs and some WRs are known to show infrared excess associated with circumstellar dust. The LBV phase is short-lived and unstable, with irregular mass ejections of $10^{-5}$ to $10^{-4} M_{\odot}$ for each outburst (Humphreys \& Davidson 1994), resulting in circumstellar nebulae and associated dust. The dust in this phase is consistent with grains of differing compositions ranging over metal-, silicate-, and carbon-rich (Voors et al. 1999; Clark et al. 2003). The WR stage, characterized by massive winds (of the order $\dot{M}=10^{-5} M_{\odot} \mathrm{yr}^{-1}$ ), is divided into two main phases (Smith 1968), the WN phase, with strong spectral lines of $\mathrm{N}$ and $\mathrm{CNO}$ cycle products, and the later WC phase, with strong lines of $\mathrm{C}$ and other He-burning products. Dust formation is associated with the WC phase, consistent with the overabundance of $\mathrm{C}$, and is notably absent in the WN phase (van der Hucht et al. 2001), although recent Spitzer observations (Barniske et al. 2006) may be the first indications to the contrary. Long-term and detailed infrared photometry of WC

\footnotetext{
1 Michelson Fellow, University of Maryland, College Park, MD 20742. Current address: CTIO, Casilla 603,La Serena, Chile; jrajagopal@ctio.noao.edu.

2 Observatoire de la Côte d'Azur, Départment Gémini, UMR 6203, Nice, France.

3 NASA Goddard Space Flight Center, Greenbelt, MD 20771.

4 Observatoire de la Côte d'Azur, Départment Gémini, Avenue Copernic, Grasse, France.

5 Department of Astronomy, University of Michigan, 501 East University Avenue, Ann Arbor, MI 4809.

6 Planetary Science MC 150-21, California Institute of Technology, Pasadena, CA 91125.

7 School of Physics, Sydney University, NSW 2006, Australia.
}

stars (Williams et al. 1987, hereafter WvdHT87), has led to considerable progress in understanding the nature of the dust and its formation. The favored mechanism for dust formation in this stage is through wind-wind collision in a WR-OB binary system (Usov 1991). For the episodic dust producers WR 140 and WR 137, photometric and spectrosocopic data (Williams et al. 1990, 2001) had already indicated binarity, which has been confirmed by longbaseline interferometric observations for WR 140 (Monnier et al. 2004b). High-resolution images in the near-IR using aperturemasking techniques with the Keck telescope provide direct evidence for binarity in the case of the constantly dusty, late-type WC stars WR 98a and WR 104 (Monnier et al. 1999; Tuthill et al. 1999) and more recently, the cocoon stars in the Quintuplet cluster (Tuthill et al. 2006). Monnier et al. (2007) present a strong case for binarity for all the dusty WRs in the Keck aperture-masking sample. However, whether all dusty late-type WCs are binaries is an open question and dust formation in single WR stars has not been ruled out (Cherchneff et al. 2000).

In this paper, we present and interpret interferometric midinfrared observations of the late-type WC stars WR 95 and WR 106, the suspected LBV-WR transition star NaSt 1 (WR 122), and the LBV AG Car. The main goals of this campaign were to resolve the sizes of the dust shells and characterize the physical distribution of dust. All the objects were observed with the MIDI instrument at the Very large Telescope Interferometer (VLTI) in the 8-13 $\mu \mathrm{m}$ wavelength range, typically with a baseline of $\sim 47 \mathrm{~m}$. NaSt 1 and WR 106 were also observed at $10.7 \mu \mathrm{m}$ with a multiaperture interferometric technique using individual segments of the Keck I telescope mirror to get complementary data from baselines up to the $10 \mathrm{~m}$ diameter of the Keck mirror. In $\S \S 2$ and 3 , respectively, we describe the observations and data reduction and present the results. Section 4 details the dust models we have 
TABLE 1

MIDI OBSERVATIONS

\begin{tabular}{|c|c|c|c|c|c|}
\hline Star & $\begin{array}{c}V \\
(\mathrm{mag})\end{array}$ & $\begin{array}{c}\text { IRAS } 12 \mu \mathrm{m} \\
(\mathrm{Jy})\end{array}$ & Type & Date Observed & Calibrators \\
\hline WR 95..................... & 13.4 & 4.56 & WC 9 & $\begin{array}{l}2004 \mathrm{Jul} 30 \\
2004 \mathrm{Jul} 31\end{array}$ & $\begin{array}{l}\text { HD 177716, HD 129456, HD } 169916 \\
\text { HD 169916, HD 165135, HD } 192947\end{array}$ \\
\hline 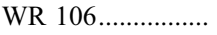 & 11.93 & 11.67 & WC 9 & 2004 Jul 9 & HD 165135 \\
\hline NaSt 1 & 15.4 & 14.5 & WN (?) & $\begin{array}{l}2004 \text { Jul } 30 \\
2004 \text { Sep } 29^{\mathrm{a}} \\
2004 \text { Sep } 30^{\mathrm{a}}\end{array}$ & $\begin{array}{l}\text { Same as WR } 95 \text { above } \\
\text { HD } 168723 \\
\text { HD } 168723\end{array}$ \\
\hline AG Car.................... & 7.09 & 12.42 & LBV & 2004 Apr 10 & HD 81797, HD 107446, HD 129456, HD 139997 \\
\hline
\end{tabular}

${ }^{\text {a }}$ Baseline UT2-UT4.

constructed and the comparison with existing models based on the spectral energy distribution. Section 5 summarizes these results and presents the conclusions.

\section{OBSERVATIONS AND DATA REDUCTION}

\subsection{MIDI}

Most targets were observed over four nights in 2004 July, except for AG Car, which was allocated one night in 2004 April (Table 1). NaSt 1 was also observed on two nights in 2004 September. All observations were with the $\sim 47 \mathrm{~m}$ long UT2-UT3 baseline, except for the 2004 September observations of NaSt 1 , which used the $\sim 89 \mathrm{~m}$ UT2-UT4 baseline. The MIDI instrument (Leinert et al. 2003) on the VLTI combines the light from a given pair of $8.2 \mathrm{~m}$ telescopes (UTs) and produces spectrally dispersed fringes in the $8-13 \mu \mathrm{m}$ band. A detailed description of the observing technique and data reduction can be found in Leinert et al. (2004). Here we limit ourselves to a brief summary.

The observing sequence starts with acquiring the target on each individual telescope with the $8.7 \mu \mathrm{m}$ filter (1.4 $\mu \mathrm{m}$ wide) and attaining maximal overlap of the two images, while chopping the secondary at $\sim 2 \mathrm{~Hz}$ over $10^{\prime \prime}$. The images are combined to produce the two complementary interferograms and dispersed through a prism with a resolution of $\lambda / \delta \lambda \sim 30$. The delay lines are scanned to find and track the fringes, and fringe visibilities are recorded while stepping the delay through $\sim 10$ wavelengths $(\lambda=10 \mu \mathrm{m})$ in steps of $2 \mu \mathrm{m}$ with a piezo-driven mirror. The scan rate is tuned to the timescale of instrumental and atmospheric fluctuations of delay, and a typical measurement records $\sim 200$ scans in about 5 minutes. After the fringe measurement, a photometric measurement is carried out by blocking the light from one and then the other telescope, while chopping the secondary mirror. No chopping is done during the fringe-tracking scans. The pointing is maintained by the Coude guiding with only tip-tilt correction; higher order adaptive optics (AO) were yet to be integrated into the system. A sensitivity better than $\sim 1 \mathrm{Jy}$ is achieved for this mode of fringe measurement. Given that these observations were made during the first regular semester after MIDI commissioning, the accuracy that we achieve for the visibility measurements is of the order of $10 \%$ and is mostly set by photometry, guiding (overlap of the two images), and fringetracking errors, which impose limits on the accuracy of calibration. We calculate the errors on the visibility as the standard deviation over the multiple calibrations for each measurement and conservatively quote the higher of this number or $10 \%$ of the visibility amplitude. The above is true for the absolute visibility amplitude errors; the relative errors are less, and the slope of the visibility in the $8-13 \mu \mathrm{m}$ band is accurate to $\sim 3 \%$.
Standard calibrator stars of known diameter from the MIDI catalog ${ }^{8}$ were observed immediately before and after the target (Table 1). Given the relatively large systematic errors inherent in the visibility determination, we have also used calibrators from other programs within a span of $2-5 \mathrm{hr}$ (depending on atmospheric conditions).

The visibility reduction method generally follows the now well-established algorithms for optical/IR interferometry (e.g., Coude Du Foresto et al. [1997] for fiber stellar interferometers; the basic principles apply for nonfiber systems like MIDI) and was carried out using custom software provided by the VLTI consortium written in the IDL language. The spectra are extracted with a typical binning of 4 pixels, each pixel being $\sim 0.05 \mu \mathrm{m}$ wide. The two complementary outputs of the combiner are differenced to remove the incoherent flux, including overall variations in the contribution from the two telescopes. Next, the fringe power is estimated from the Fourier transform, and the visibility amplitude, ${ }^{9}$ normalized by the photometric fluxes, is calculated. The visibility is then calibrated using the instrument visibility measured on the calibrator stars.

\subsection{Keck}

We carried out observations at $10.7 \mu \mathrm{m}$ with the Keck I telescope on two stars in our sample, WR 106 and NaSt 1, in 2005 May (Table 2). These observations were carried out in a segmenttilting mode (Monnier et al. 2004a; Weiner et al. 2006) to achieve close to diffraction-limited resolution. Imaging at $10 \mu \mathrm{m}$ with the full pupil has, in practice, failed to reliably yield diffractionlimited images. This is in part attributable to the seeing spike problem, in which the visibilities at shorter baselines are difficult to calibrate in the presence of changes in the seeing between the

8 See http://www.eso.org/projects/vlti/instru/midi/midi_calib.txt.

9 The usual estimator used in optical/infrared interferometry is the square of the visibility amplitude $\left(V^{2}\right)$, since this quantity can be corrected for detector noise bias. However the MIDI measurement error in the mode we used (HighSens, with no simultaneous photometry) is dominated by calibration error, not detector noise.

TABLE 2

Keck OBservations

\begin{tabular}{|c|c|c|}
\hline Star & Date of Observation & $\begin{array}{c}\text { Gaussian FWHM (at } 10.7 \mu \mathrm{m} \text { ) } \\
\text { (mas) }\end{array}$ \\
\hline WR $106 \ldots \ldots \ldots \ldots . . . .$. & 2005 May 26 & $56.0 \pm 6.9^{\mathrm{a}}$ \\
\hline NaSt 1 ............... & 2005 May 26 & $50.0^{\mathrm{b}}$ \\
\hline
\end{tabular}

a Dominant error arises from calibration.

b Object was unresolved. Value is an estimated upper limit. 

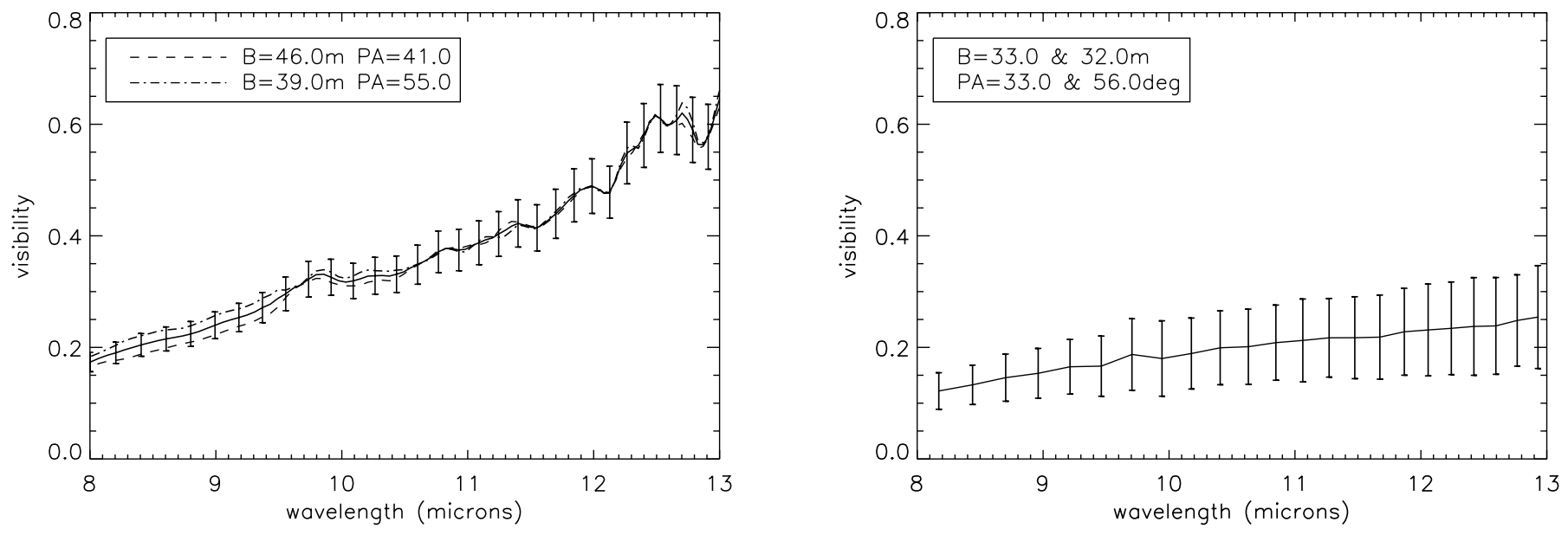

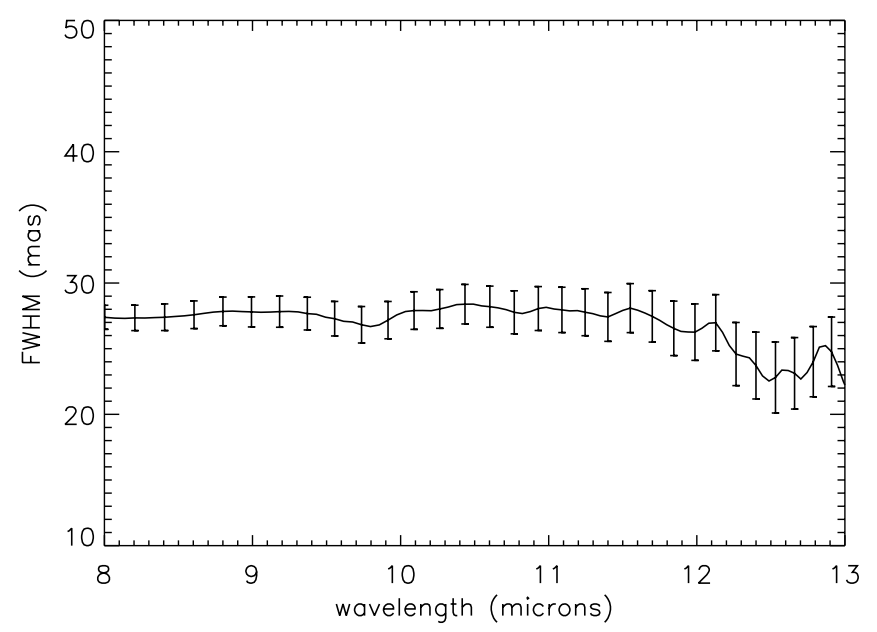

FIG. 1.-Top: Visibility profile with wavelength for WR 95 . The solid line is the mean of the two measurements. Bottom: Corresponding (mean visibility) Gaussian FWHM

target and the calibrator. The results shown here demonstrate the utility of this new method in alleviating some of these problems. In this technique, custom software is used to reconfigure the segmented primary mirror to allow selected groups of segments to focus on the detector (the Long-Wavelength Spectrograph camera). Each group (four, in our case) of six segments is chosen to form a nonredundant sparse-aperture array. The $1.8 \mathrm{~m}$ hexagonal segments are individually phased up so that each group focuses on a separate spot on the detector. Thus in a given frame, 24 out of 36 segments are used in these four Fizeau arrays (the rest of the segments are pointed away from the detector). This arrangement measures 15 baselines, ranging from about 2 to $9 \mathrm{~m}$, and 20 closure phases. Each frame is of the order of $10 \mathrm{~ms}$, and we co-add nine frames, giving an effective exposure of $90 \mathrm{~ms}$. Chopping is done at a $5 \mathrm{~Hz}$ rate. Calibrators are observed before and after the targets, and each target is visited at least twice during a night. The analysis of the data closely follows the aperture-masking analysis described in detail in numerous publications (e.g., Tuthill et al. 2000). These measurements complement the long-baseline VLTI data by adding in visibilities at shorter baselines up to $9 \mathrm{~m}$.

\section{RESULTS}

\subsection{The WC Stars}

The two WC type stars in our sample are WR 95 and WR 106. They are both classified as late-type (WC 9) and are known to be

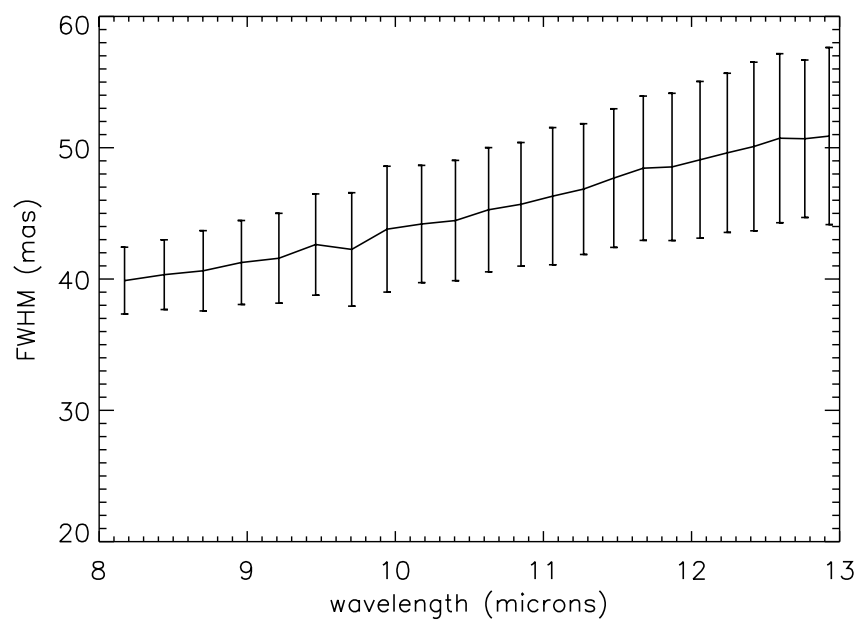

FIG. 2.-Top, Visibility profile with wavelength for WR 106; bottom, corresponding Gaussian FWHM.

persistent dust producers from IR excess detected over long-term photometry (Williams 1995). The photometric data do not have any periodicity, nor do the spectra indicate any clear signs of binarity. We obtained two MIDI visibility measurements on WR 95 on consecutive nights in 2004 July. Although the projected baseline and position angle differ slightly between these two measurements, the visibility values were remarkably similar (Fig. 1, broken lines). Although this might indicate a small degree of asymmetry, the accuracy of these measurements is not high enough to justify this interpretation. Instead, we have chosen the average of the two sets to represent the spectrally dispersed visibility (Fig. 1, solid line). As can be seen in Figure 1, we clearly resolve the WR 95 dust shell with visibility values ranging from $\sim 0.2$ to $\sim 0.6$ over the $8-13 \mu \mathrm{m}$ band. In the absence of detailed knowledge of the source geometry, we have assumed a Gaussian profile, and the resulting FWHM in milliarcseconds (vs. wavelength) is shown in Figure 1 (right). WR 95 was not observed with the Keck segment-tilting method, since it was below the sensitivity limit.

Figure 2 shows the MIDI results for WR 106. Although we obtained two measurements in July 2004 (see Table 1), poor seeing conditions resulted in variations in fringe tracking and calibration between the two observations. In the absence of a clear criterion to select one data set over the other, we average the two, and the resulting larger error bars are shown in the figure. However, this target is well resolved, and meaningful conclusions can be 
TABLE 3

Measured Sizes (MIDI)

\begin{tabular}{|c|c|c|c|c|}
\hline Star & $\begin{array}{c}\text { Distance } \\
(\mathrm{kpc})\end{array}$ & $\begin{array}{c}\text { Gaussian FWHM } \\
\text { at } 10.5 \mu \mathrm{m} \\
\text { (mas) }\end{array}$ & $\begin{array}{l}\text { Linear Size } \\
(\mathrm{AU})\end{array}$ & References \\
\hline WR $95 \ldots \ldots \ldots . . .$. & 2.09 & $28.4 \pm 1.5$ & 59.4 & 1 \\
\hline WR $106 \ldots \ldots . .$. & 2.3 & $45.0 \pm 5.0$ & 103.5 & 1 \\
\hline NaSt 1 .............. & 2.0 & $20.1,14.8( \pm 2.0)^{\mathrm{a}}$ & $40.2,29.6$ & 2 \\
\hline AG Car........... & 6.0 & $12.5 \pm 3.0$ & 75 & 3 \\
\hline
\end{tabular}

a The two values are from two different baselines. Interpreting sizes from visibility may be inaccurate for this object; see text.

References.-(1) van der Hucht 2001; (2) Crowther \& Smith 1999; (3) Humphreys et al. 1989.

drawn, even from the less than optimum data. The visibility variation with wavelength is similar to that for WR 95 .

WR 106 was resolved by the Keck single aperture (Table 2). This was surprising in that, given the visibility values measured by the VLTI, one would have expected an unresolved source at the comparatively short baselines offered by the Keck mirror. This indicates that there is an extended component in the dust distribution that was overresolved and hence failed to show up in the VLTI observations. We present these results in $\S 4$, where we discuss some simple models.

In Table 3, we list the angular sizes (Gaussian FWHM) measured with MIDI at $10.5 \mu \mathrm{m}$ and the corresponding linear sizes of the objects, assuming distances from the literature (see table footnote). The parameters directly constrained by this data set are the size of the dust shells and its variation with wavelength. The radius at which the dust grains start to condense is a much debated and crucial topic in the theory of dust formation around WR stars (Zubko 1998; Crowther 2003). The major difficulty has been that the equilibrium temperatures at the radii predicted by the SEDbased models are often above the sublimation temperature for carbon dust. Dust formation in the shock interface of a collidingwind system has found some favor in this context. A recent summary of results from a long-term campaign of aperture-masking interferometry at the Keck (Monnier et al. 2007) provides the near-IR sizes for a number of WR stars, including those in our sample. The authors point to a tight correlation between the surface brightness in the 1.5-3.0 $\mu \mathrm{m}$ band and the sizes (Gaussian FWHM) of the objects in their sample to argue for a common underlying dust production mechanism. Our direct measurement of the mid-IR size scale for these objects is an important addition to this debate. From our Gaussian FWHM curves, it is clear that the size of the emitting region is more or less invariant in the $8-13 \mu \mathrm{m}$ band. This could indicate that the emission is dominated by an inner edge or ring of material. In fact, the sizes for WR 95 at the shorter wavelengths $(1.6$ and $2.2 \mu \mathrm{m})$ reported in Monnier et al. (2007) are quite similar to our values. For WR 106, the mid-IR size is larger, as expected from the presence of the extension seen in the Keck measurement. In $\S 4$, we present radiative transfer models for the dust shells based on our measurements and derive inner edge radii, which we compare with values from SED-based models.

\subsection{NaSt 1}

NaSt 1 has a checkered history of classification. It was initially typed as a late-type WN star, WR 122 (van der Hucht et al. 1981), but subsequently this classification has been questioned. The most recent study (Crowther \& Smith 1999) has resulted in this star not being considered a Wolf-Rayet any longer. Crowther and Smith find the chemical composition of the optical nebulosity
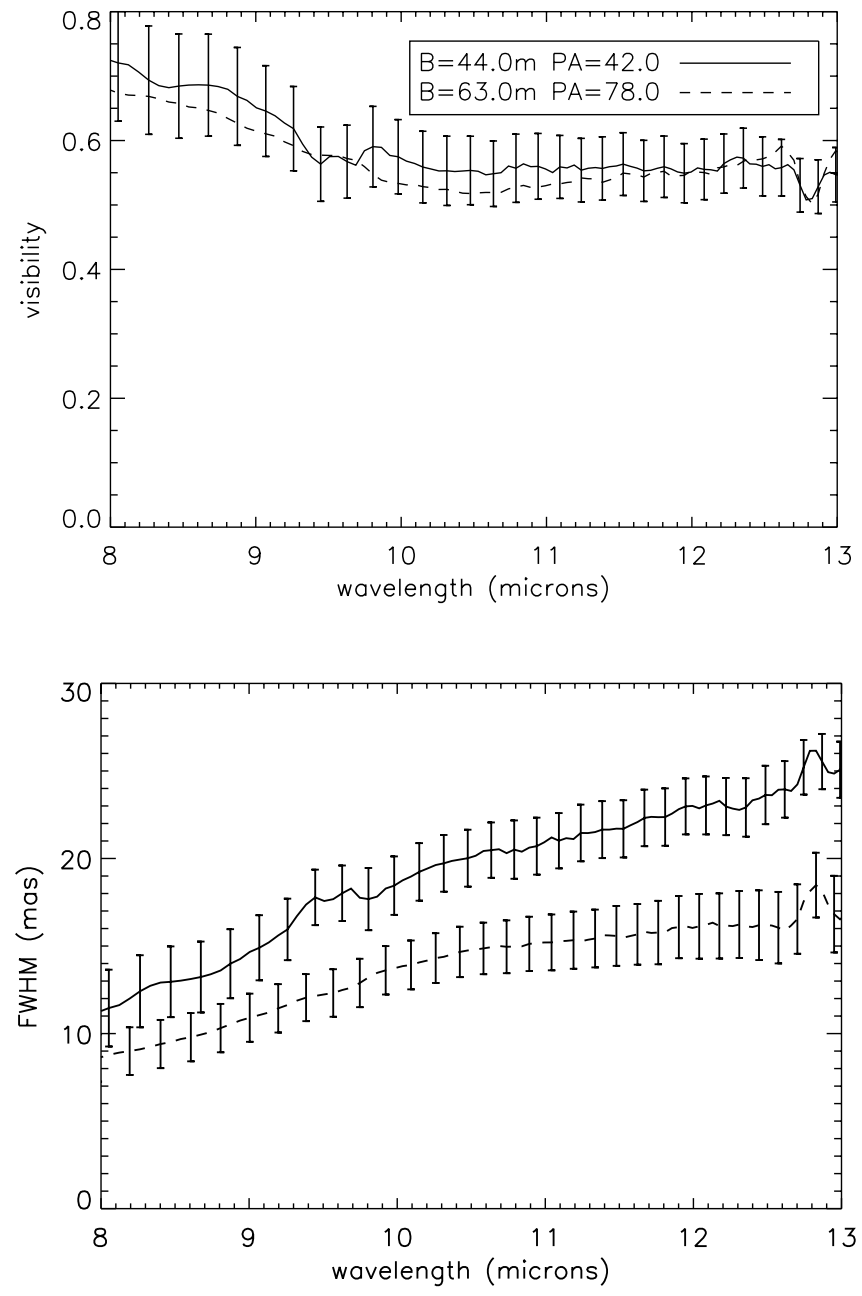

FIG. 3.-Top: Visibility profile with wavelength for NaSt 1 (only one set of error bars shown for clarity). Bottom: Corresponding Gaussian FWHM. Transforming the visibility directly to a Gaussian size is, however, probably inaccurate for this object (see text).

surrounding the star to be similar in many respects to an LBV, such as $\eta$ Carina, and conjecture that the obscured central star could be an early-type WN, presumably just leaving the LBV phase. The uncertainty in classification is in part due to the rarity of these very massive stars, especially the one caught in transition. As such, our measurement of the extent of the close-in dust and its variation with wavelength is significant. The source is unresolved by MIDI in single-dish imaging mode, and the flux measured within the $\sim 300$ mas beam is similar to the value quoted in Smith \& Houck (2001, Fig. 4 in their paper) from a bigger beam, indicating that all the mid-IR emission originates from a compact object. Another notable feature is the absence (as in the WCs) of any silicate feature in the visibility profile (Fig. 3) or the MIDI spectrum (Fig. 4). Although this does not rule out the presence of silicate dust, it attests to the fact that there are no structures with silicate emission at the MIDI resolution scale. This is in agreement with the finding of Crowther \& Smith (1999) that the nebula is highly enriched in CNO-processed material.

NaSt 1 was not resolved with the Keck single aperture. In our experience, the segment-tilting method is capable of resolving sizes down to $40-50$ mas at the $3 \sigma$ level. At $10.7 \mu \mathrm{m}$, this is approximately a factor of 6 better than the formal diffraction limit $(1.22 \lambda / D)$ for the $10 \mathrm{~m}$ aperture. A more rigorous analysis for the resolution limit for aperture-masking observations at Keck (the near-infrared analog of segment-tilting) estimates it to be 4 times 


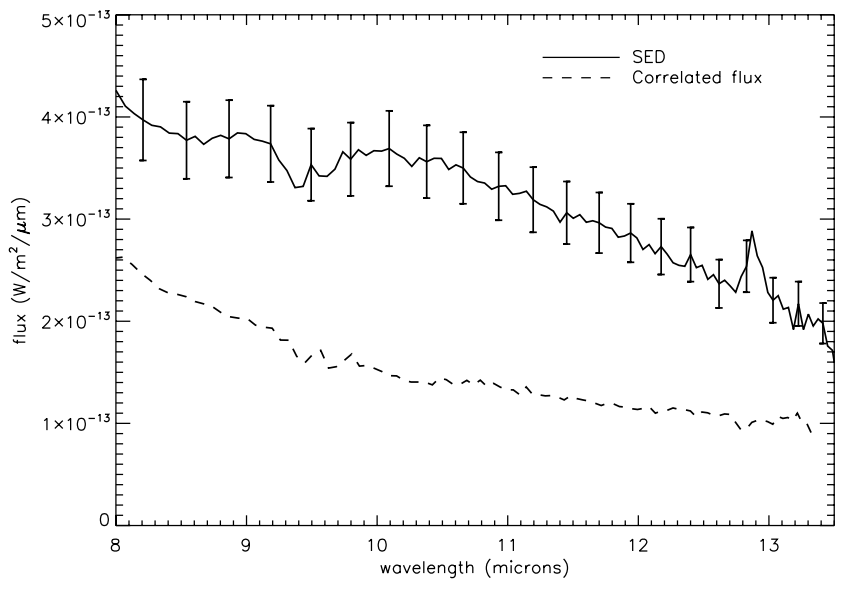

FIG. 4.-MIDI spectrum and correlated flux for NaSt 1. The [Ne II] $12.8 \mu \mathrm{m}$ feature is not seen in the correlated flux and is likely fully resolved by the interferometer, as is a large fraction $(\sim 1 / 2)$ of the total continuum flux.

better than the diffraction limit (Monnier et al. 2007). We estimate an upper limit of 50 mas for NaSt 1 from the Keck experiment.

NaSt 1 was observed with MIDI at both the UT2-UT3 (projected baseline $44.0 \mathrm{~m}$, P.A. $42^{\circ}$ ) and the longer UT2-UT4 (projected baseline 63.0 m, P.A. $78^{\circ}$ ). Figure 3 shows the visibility values and corresponding Gaussian sizes for NaSt 1 . The visibility varies with wavelength, decreasing toward the red end of the spectrum. This typically indicates cooler material farther away from the star and is in contrast to the behavior we see in the WC stars. At least part of the dust around LBVs could be swept from the interstellar medium (ISM) and/or formed in a cooler environment in the episodic ejecta from the LBV phase and could lead to a less defined inner edge and more spread in the dust temperature. The visibility on the P.A. $42^{\circ}$ shorter baseline is very similar to that on the longer one, and, if we interpret this directly as a size, may indicate an elongation in this direction. The optical image of NaSt 1 (Crowther \& Smith 1999) in the N II $\lambda 6583$ line shows an elliptical nebula with the major axis at a P.A. of $30^{\circ}$ and an aspect ratio of 0.6 , although the size scale $\left(\sim 8^{\prime \prime}\right)$ is several orders of magnitude larger than the milliarcsecond scales we measure.

However, it must be stressed that the visibility curves from the two different baselines are almost identical, and the probability of obtaining the same profile for a spatially complex object (even if as simple as an ellipse) by pure chance is very low. A possible alternative interpretation is that, following the suggestion of Crowther \& Smith (1999) the mid-IR source could be split into two components: a compact, unresolved part, with a correlated flux of about $5 \mathrm{Jy}$ from 8 to $13 \mu \mathrm{m}$, corresponding to the central source and perhaps some hot dust in its immediate surroundings, and a detached $\eta$ Carina-like nebula, fully resolved by the interferometer. The extension of this nebula may be of the same order as that of the region contributing the [Ne II] $12.8 \mu \mathrm{m}$ line, which is seen in the single-dish spectrum, but not in the correlated (i.e., arising in the unresolved core) flux (Fig. 4). However, the upper limit on the size of this component would be limited to 50-60 mas at most; anything bigger would have been resolved by the Keck observations. Given the $10 \%$ error on the MIDI visibilities, this could still be overresolved, although marginally, even at the shorter $44 \mathrm{~m}$ baseline. The size of the compact component is possibly less than 5 mas or so, given that higher values would start to affect the relative slope of the visibilities between the two baselines. Qualitatively, this hypothesis offers a natural explanation of the measurements: the central source is unresolved and the extension overresolved, whatever the baseline length ( 44 or
$63 \mathrm{~m})$. However, the tight constraints on the extended component weaken the case. Clearly, more observations are needed to clarify the picture. A very hot source $(T \sim 100,000 \mathrm{~K})$ is required to fit the object's SED (spectral energy distribution) by Crowther $\&$ Smith (1999), and the $5 \mathrm{Jy}\left(1.5 \times 10^{-13} \mathrm{~W} \mathrm{~m}^{-2} \mu \mathrm{m}^{-1}\right)$ correlated flux we measure at $10 \mu \mathrm{m}$ is not consistent with this. A constant correlated flux from 8 to $13 \mu \mathrm{m}$ (Fig. 4) can hardly be attributed to a source at a single temperature (say, $\sim 2000 \mathrm{~K}$ ) either. In order to decompose the SED into multiple sources at different temperatures, it is important to study this object in the near-IR using the AMBER instrument at the VLTI to resolve the $K$-band continuum and the very strong He I $2.058 \mu \mathrm{m}$ line, which may originate in the close vicinity of the star (Crowther \& Smith 1999, their Fig. 6).

At a distance of $2 \mathrm{kpc}$, the maximum linear size from the Gaussian model is $\sim 40 \mathrm{AU}$, and with a two-component model it is $\sim 100 \mathrm{AU}$ (for comparison, the average extension of the optical nebula is $\sim 13,600$ AU at $2 \mathrm{kpc}$ from Crowther \& Smith). If we take the same characteristic expansion velocity as Crowther $\&$ Smith $\left(15 \mathrm{~km} \mathrm{~s}^{-1}\right)$, then the dynamical age of the dusty nebula at $100 \mathrm{AU}$ from the source is about $30 \mathrm{yr}$. This shortens to a couple of years if instead we assume dust grains forming in the wind at a velocity of $\sim 100-300 \mathrm{~km} \mathrm{~s}^{-1}$ (Zubko 1998; Crowther \& Smith 1999). Hence, the dust that we resolve points to very recent or ongoing dust formation close to the star, which has implications for its current poorly known evolutionary state.

\section{3. $A G C a r$}

AG Car is one of the best studied LBVs and a prototype of its class. AG Car has been extensively observed from UV to radio wavelengths with photometric, spectroscopic, and polarimetric techniques over several decades (Humphreys et al. 1989), which locates it in the H-R diagram very close to the HumphreysDavidson limit, an empirical luminosity boundary for evolved massive stars (Humphreys \& Davidson 1979).

AG Car is surrounded by a bright extended bipolar nebula, $30^{\prime \prime} \times 40^{\prime \prime}$ in size (Nota et al. 1992), perhaps from a massive eruptive event $10^{4}$ yr ago (Lamers et al. 2001). The kinematics of the bipolar nebula is consistent with a two-lobe polar ejection viewed nearly from the equator (Nota et al. 1992). Spectroscopic analysis by Groh et al. (2006) supports the bipolar geometry and shows the $50-100 R_{\odot}$ supergiant central star to be rotating close to its breakup velocity. The high rotational velocity after the huge LBV outburst may be understood if the original explosion expelled the mass and momentum preferentially toward the poles. The wind at smaller latitude may not have formed or may have launched material with velocities lower than the escape velocity, which fell back onto the star. The nebula contains a large amount of dust (Voors et al. 2000; 0.2 $M_{\odot}$ ), which implies a huge massloss rate during the eruption (at least $8-15 M_{\odot}$ ).

Our measurements are set in context by two studies in particular, Voors et al. (2000) and Nota et al. (2002). The images in Voors et al. (2000) obtained with the $10 \mu \mathrm{m}$ TIMMI camera on the $3.6 \mathrm{~m}$ NTT at La Silla reveal a central peak and a detached nebula with a radius of $\sim 10^{\prime \prime}$. Their SED analysis reveals a complex situation: the dust is dominantly oxygen-rich, but polycyclic aromatic hydrocarbon (PAH) features are observed at 3.3, 7.7, 8.6, and $11.3 \mu \mathrm{m}$, revealing some amount of carbon dust. Moreover, a population of very large grains $(>10 \mu \mathrm{m})$ is necessary to explain the flux levels at long wavelengths, whereas a population of small warm grains is invoked to explain the flux between 5 and $20 \mu \mathrm{m}$.

There is evidence for $\mathrm{CO} J=1 \rightarrow 0$ and $2 \rightarrow 1$ emission associated with AG Car (Nota et al. 2002), and the authors conclude 

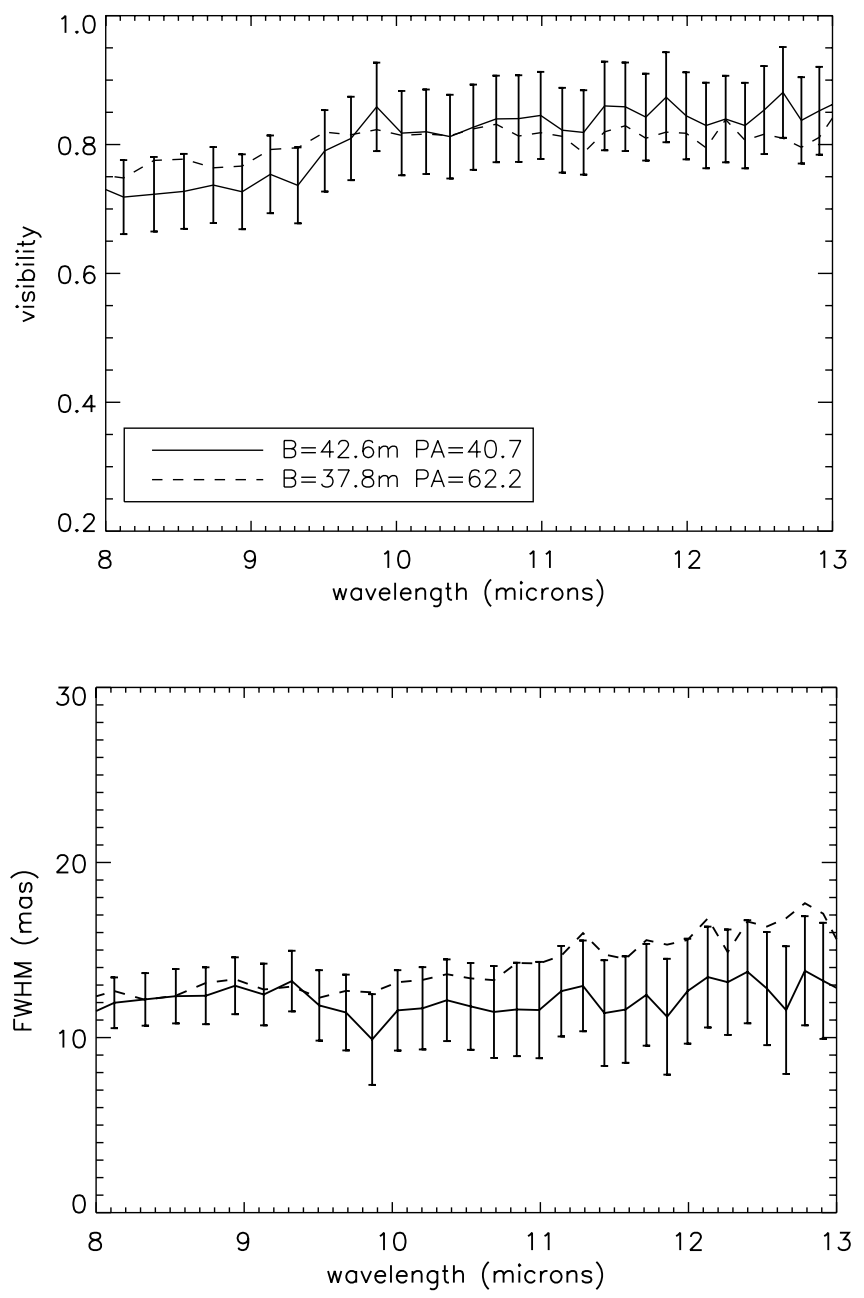

FIG. 5.-Top: Visibility profile with wavelength for AG Car. Bottom: Corresponding Gaussian FWHM. The error bars are similar for both measurements. For clarity, only one set is shown.

that the unresolved $\mathrm{CO}$ emission must arise close to the star in a high-density warm region and not in the detached nebulae resolved in optical emission-line images. They postulate that a warm and dense equatorial disk exists close to the star.

MIDI observations of AG Car were carried out with the UT2UT3 baseline on two nights, with projected baselines of 42.0 and $35.6 \mathrm{~m}$ and P.A. of $41^{\circ}$ and $69^{\circ}$, respectively. The visibilities and corresponding Gaussian sizes are shown in Figure 5 and the spectrum in Figure 6. AG Car is marginally resolved. The measured size $(\sim 11$ mas $)$ is similar for both observations and fairly uniform with wavelength. The Nota et al. (2002) optical images show strongly enhanced $\mathrm{H} \alpha$ brightness at P.A. $\sim 35^{\circ}-225^{\circ}$, and the morphology is similar at $12 \mu \mathrm{m}$ (Voors et al. 2000). This direction is also the waist of the nebula, the largest extension being seen perpendicular to it. The MIDI baselines are therefore oriented close to an optimal direction to detect equatorial material, and we are very likely resolving the equatorial disk, although we cannot at present distinguish between a disk and shell structure. This is the first direct evidence for dust in a compact structure. The size we detect (11 mas) is $\sim 3$ orders of magnitude smaller than the upper limit $\left(3^{\prime \prime}\right)$ on the size of the (unresolved) $\mathrm{CO}$ outflow in Nota et al. (2002). The corresponding linear size is of the order of only $100 \mathrm{AU}$ at a distance of $6 \mathrm{kpc}$, very close to the central star. Nevertheless, this detection adds weight to the Nota et al. (2002) claim that the $\mathrm{CO}$ emission arises from molecular gas close to the

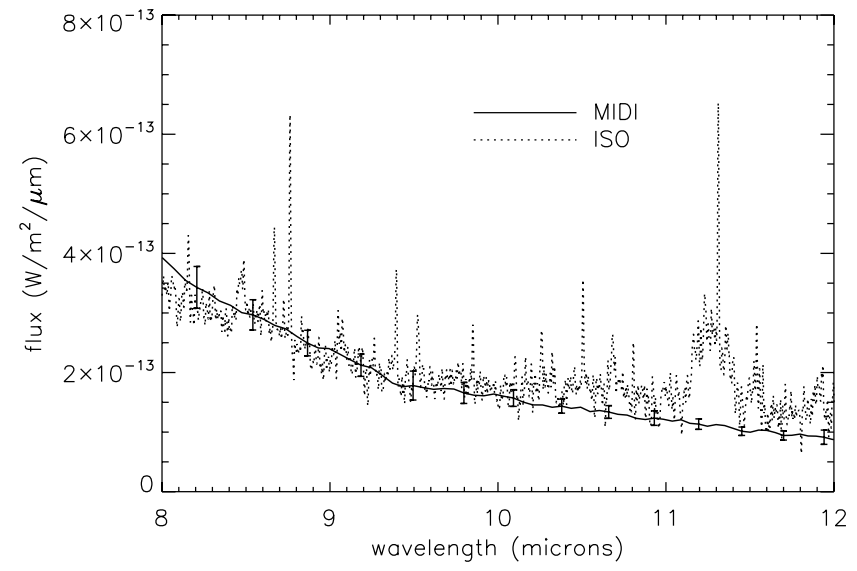

FIG. 6.- MIDI spectrum for AG Car, with the $I S O$ spectrum (scaled overall to match the MIDI flux level) for comparison. The PAH feature at $11.3 \mu \mathrm{m}$ is not seen in the MIDI spectrum.

star and is shielded by a disk structure. The presence of dust has implications also for the origin of this compact structure. Nota et al. (2002) favor a scenario in which the star goes through a red supergiant (RSG) or RSG-like phase (Smith et al. 1998; Lamers et al. 2001) during which the molecular outflow is formed. Dust formation is also commonly observed during the RSG stage (Massey et al. 2005; Danchi et al. 1994) and fits in with this hypothesis, although the $\sim 100$ AU scale size indicates very recent or current dust formation as well.

There are few published spectra of AG Car in the mid-IR, and the MIDI spectrum is a valuable addition. For comparison, we also show in Figure 6 the spectrum from the Short Wavelength Spectrometer (SWS) on the Infrared Space Observatory (ISO) in the $8-13 \mu \mathrm{m}$ range (Sloan et al. 2003), scaled to roughly match the flux level of the MIDI spectrum. The ISO spectrum, with a much bigger aperture, exhibits a slightly different slope from the MIDI measurement, but the striking difference is that the MIDI spectrum does not show the $11.3 \mu \mathrm{m}$ PAH feature, implying that the PAH originates from farther out in the disk/shell, as conjectured by Voors et al.

\section{THE WC MODELS}

The dust around late-type WR stars has been closely studied over the last two decades or so and presents a challenging problem pertaining to its formation, geometry, and evolution. There have been quite a few attempts to model the dust in some detail. WvdHT87 model these as spherical shells based on extensive IR spectroscopy. Zubko (1998) provides dust models with emphasis on the physics of grain formation and growth, taking into account the grain dynamics in the stellar wind. Harries et al. (2004) take advantage of the high-resolution near-IR images of WR 104 (Tuthill et al. 1999), which show a pinwheel of dust, formed in a binary wind interface, to construct a three-dimensional radiative transfer model for this object. As mentioned earlier, a large percentage of the known dust-forming late-type WC stars are known to be binaries, WR 104 being a prime example. The formation of dust in these stars is usually interpreted using the wind-wind collision model of Usov (1991). None of the stars in our sample show definite evidence of being binaries (Williams \& van der Hucht 2000), although this remains a strong possibility.

It has been difficult to explain dust formation in the hostile environment of the wind from single WR stars. SED-based models typically have dust forming at distances from 300 up to 1000 times the stellar radius (Zubko 1998). The consensus on the 
TABLE 4

Starting Parameters for the WC Models

\begin{tabular}{|c|c|}
\hline Parameter & Value $^{a}$ \\
\hline$T_{\text {eff }} \cdots \cdots \cdots \cdots \cdots$ & $1900 \mathrm{~K}$ \\
\hline 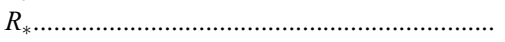 & $14.6 R_{\odot}$ \\
\hline 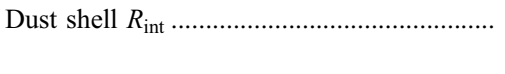 & $\begin{array}{c}410 R_{*}(\text { WR } 95) \\
280 R_{*}(\text { WR 106) }\end{array}$ \\
\hline 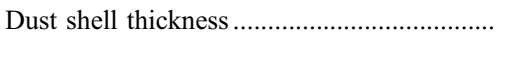 & $\begin{array}{c}3 R_{\text {int }}(\text { WR } 95) \\
3 R_{\text {int }}(\text { WR 106) }\end{array}$ \\
\hline 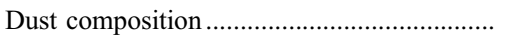 & Amorphous C \\
\hline Density profile & $r^{-2}$ \\
\hline 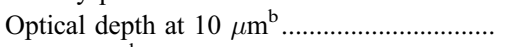 & 0.01 \\
\hline 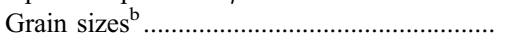 & $0.1-1 \mu \mathrm{m}$ \\
\hline
\end{tabular}

Note.-Unless stated otherwise, all values are from WvdHT87.

a Single values are common for both stars.

b See text, $\S 4.2$, for references.

material of the dust, from the SED signature, is amorphous carbon (WvdHT87). However, at these distances, the electron temperatures in the winds of WRs are likely as high as $10^{4} \mathrm{~K}$ (Zubko 1998), implying that the carbon is almost fully ionized.

We present here radiative transfer models for the WC stars in our sample with an aim to use the interferometric measurements to answer the crucial question about the formation radius of the inner edges of these dust shells. To this end, we use a twodimensional Monte Carlo radiative transfer code, MCTRANSF (Niccolini et al. 2003), to construct spherically symmetric dust distributions consistent with the measured visibilities.

The parameters of the models are the star diameter and effective temperature, the internal and external radius of the envelope, its density profile and optical depth, the minimal and maximal radius and size distribution of the grains, and the optical indices of the dust. We start with the basic parameters for the dust shells around WR 95 and WR 106, mostly taken from the comprehensive SED-based models of WvdHT87 (see Table 4). By propagating energy packets in the sampled envelope (circular grid), taking into account the absorption, emission, and scattering characteristics of the dust, MCTRANSF computes the temperature of the envelope, the SED of the flux received by the observer directly from the star, and the flux emitted and scattered by the envelope. The code has been described in detail in Niccolini et al. (2003; see Pascucci et al. [2004] for benchmarking tests). An example application in modeling a circumstellar dust shell is found in Woitke \& Niccolini (2005).

We then explore the parameter space around this model by primarily varying the location of the inner edge of the disk to see what values are most consistent with both our measured visibilities and spectra. We also tried limited variations of the optical depth, grain size, and distribution to quantify their effect on the visibility profile and spectra. The radius of the central star and the composition of the dust were not changed in these trials. MCTRANSF does not explicitly incorporate the sublimation temperature of the grain as an input. The dust temperature in the nucleation zone from existing observational and modeling constraints ranges from 1000 to $1500 \mathrm{~K}$ or so (Zubko 1998; Harries et al. 2004), and in the process of iteration we discard those models that produce untenably high temperatures at the inner edge of the shell. We stress that the modeling is not an exercise in fitting. That does not seem justified, given the limited visibility data and the fact that the actual geometry of the dust is most likely more complex than simple spherical shell models. Our goal here is to use the new measurements to constrain the vitally important distance to the dust-forming zone from the star.

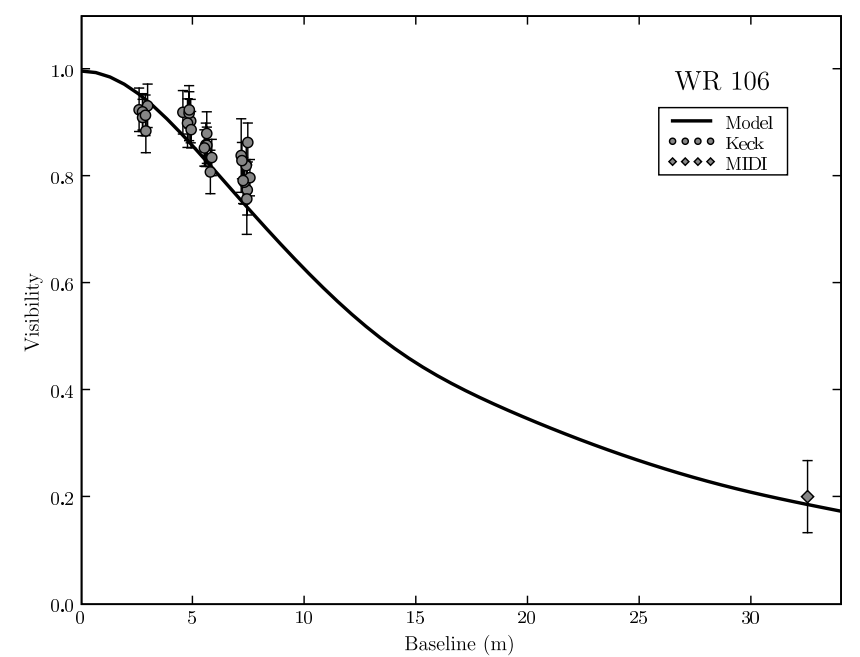

FIG. 7.-Visibility vs. baseline for WR 106. The solid line represents the model visibility, the filled circles represent the Keck visibilities (upper left), and the diamond represents MIDI at $10.5 \mu \mathrm{m}$.

\subsection{WR 106}

Extensive studies of the SED of this well-known dust-producing WC9 star exist in the literature, and long-term IR photometry is summarized in van der Hucht et al. (2001). Infrared ( $J-Q$ bands) measurements are presented in WvdHT87. More recent mid-IR $I S O$ spectra are found in Smith \& Houck (2001). Given the difficulty in standardizing mid-IR photometry from various sources and the fact that dusty WC stars can have differing degrees of variability, we rely mostly on the $8-13 \mu \mathrm{m}$ spectra obtained with the MIDI instrument simultaneously with the visibilities. Leinert et al. (2004) have confirmed the reliability of spectra from MIDI. We used standard reduction techniques such as they describe to extract the spectra; most of our interferometric calibrators were also chosen to be IR spectroscopic standards ${ }^{10}$ and as such provide ideal templates to calibrate the spectra. Dereddening WR spectra has been nontrivial, primarily because these stars are grouped toward the Galactic center, with large columns of intervening interstellar dust in addition to the circumstellar dust. The wide interstellar silicate feature centered around $9.7 \mu \mathrm{m}$ complicates the issue (Smith \& Houck 2001). To provide a uniform comparison, we have dereddened the $8-13 \mu \mathrm{m}$ band with values of $A_{\lambda} / A_{v}$ from WvdHT87 and the van der Hulst extinction curve 15 for the shorter wavelengths. For WR 106, the model visibilities from our "best" model are shown in Figure 7, along with the Keck measurements, as well as the MIDI visibility at $10.5 \mu \mathrm{m}$.

The MIDI visibilities across the entire band $(8-13 \mu \mathrm{m})$ are compared with the model in Figure 8. The closure phases measured by the Keck segment-tilting experiment did not show any significant deviation from zero, signifying a lack of asymmetry at this resolution and sensitivity. We therefore plot the Keck visibilities, treating the baselines as scalars (i.e., ignoring the orientation). Figure 9 shows the model SED, along with our measurements and those from the literature, ${ }^{11}$ spanning wavelengths

10 See http://ssc.spitzer.caltech.edu/irs/calib/templ/cohen_models.

11 From catalogs available at the VizieR online database (Ochsenbein et al. 2000). Optical fluxes: Homogeneous Means in the UBV System (Mermilliod \& Mermilliod 1994); Hipparcos Input Catalogue, Version 2 (Turon et al. 1993); the USNO-B1.0 Catalog (Monet et al. 2003); IR fluxes: Catalog of Infrared Observations, Edition 5 (D. Y. Gezari, P. S. Pitts, \& M. Schmitz, 1999, unpublished); Third release of DENIS, from the DENIS consortium in 2005. 


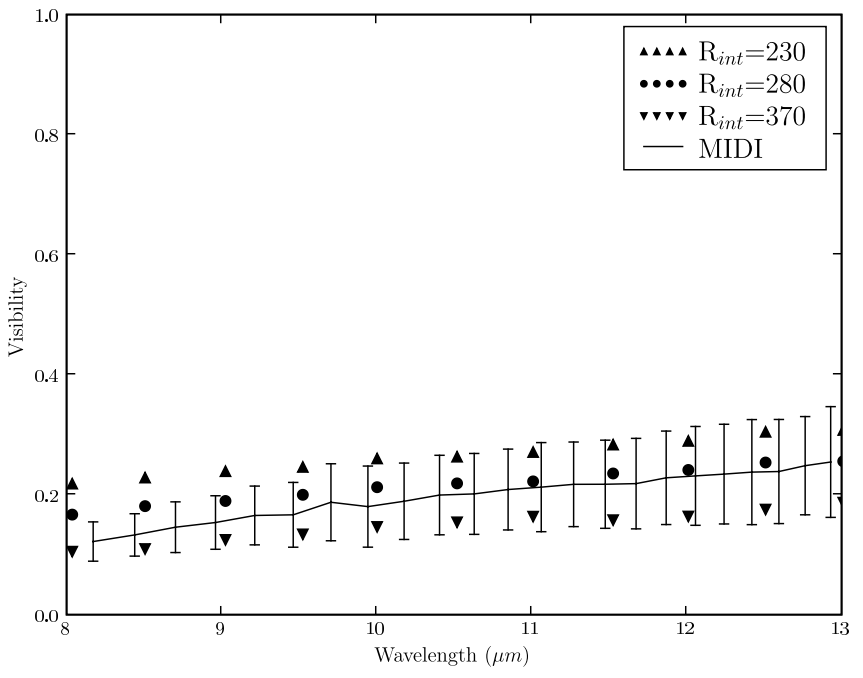

FIG. 8.-Visibility vs. wavelength for WR 106. The line (with error bars) indicates the MIDI measurement. Models with varying inner radii, $R_{\text {int }}$ (in units of $R_{*}$ ), for the shell are shown for comparison.

from the optical to the far-infrared for comparison. The temperature profile of the dust in our model is shown in Figure 10. The parameters for the model are as below.

\subsubsection{Central Star}

For the central star, we have adopted a blackbody spectrum at the effective temperature listed below. The temperature of the WR star has been assigned a range of values in the literature. Most dust formation models adopt a value between 18,000 and 25,000 K (e.g., WvdHT87). These values have been criticized as being underestimates from recent studies of the WR spectra (Crowther 2003). However, we find in our models that higher temperatures (we tested up to $40,000 \mathrm{~K}$ ) do not fit the $8-13 \mu \mathrm{m}$ spectra and also lead to dust temperatures above the $1500 \mathrm{~K}$ or so sublimation temperature usually adopted for amorphous carbon grains. This indeed has been the reason that previous modeling attempts favor the lower values as well. Although more sophisticated SEDs have been used for the stellar photosphere (Harries et al. 2004; WvdHT87), a simple blackbody is likely adequate for our pur-

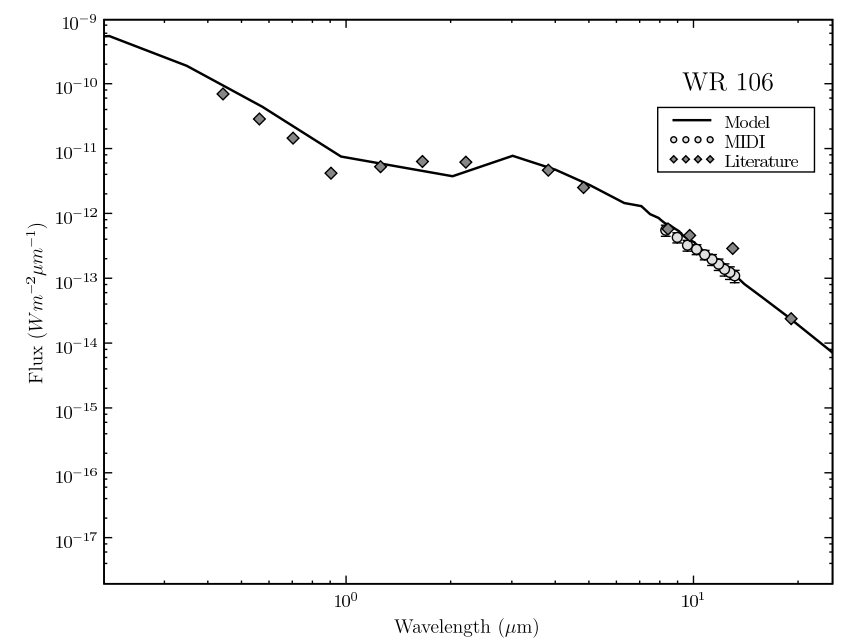

FIG. 9.- SED of WR 106, showing our best model (solid line), the measurements from MIDI ( gray circles), and the measurements from the literature (squares; see text for sources).

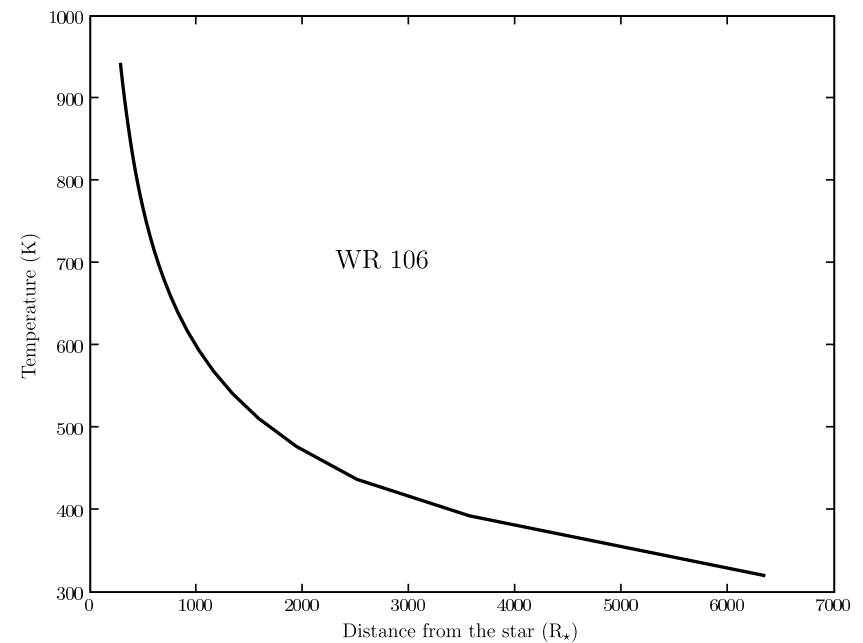

FIG. 10.-Dust temperature profile for the WR 106 best model. Distance from the star is in units of $R_{*}$.

pose. The radius of the star is assumed to be $14.6 R_{\odot}$ (WvdHT87) at a distance of $2.09 \mathrm{kpc}$ (van der Hucht 2001).

1. Temperature of central star $T_{\text {eff }}=19,000 \mathrm{~K}$.

2. Angular radius of central star $R_{*}=3.23 \times 10^{-5}$ arcsec.

\subsubsection{Dust Shell Physical Properties}

1. The composition is amorphous carbon (optical constants from Rouleau \& Martin 1991).

2. The grain size ranges from 0.5 to $0.6 \mu \mathrm{m}$.

3. The optical depth is $\tau=0.015$ at $10 \mu \mathrm{m}$.

\subsubsection{Dust Shell Geometry}

1. The inner radius of dust shell is $R_{\text {int }}=280 R_{*}$ (multiples of stellar radius).

2. The outer radius of dust shell is $8400 R_{*}$.

3 . The density profile is proportional to $r^{-2}$, except in the region between $1.0 R_{\text {int }}$ and $14.0 R_{\text {int }}$, where we introduce an overdense region with density proportional to $7 r^{-2}$.

\subsection{Discussion}

Most of the starting parameters of the dust shell model were based on WvdHT87. They establish the composition to be amorphous C, at least in the case of the episodic dust producer WR 140. The lack of silicate features in the mid-IR region also points to amorphous $\mathrm{C}$. The grain size and distribution for the shells are not well known. The Zubko (1998) grain formation model predicts a fairly small maximum grain size $(\sim 100-200 \AA)$. Harries et al. (2004) model the inner part of the WR 104 pinwheel nebula with small $(\sim 100 \AA)$ grains, mainly to match the $2-10 \mu$ m flux. However, there is evidence in the SED of WR nebulae pointing toward large grains of $\sim 0.1$ to $1 \mu \mathrm{m}$ (Chiar \& Tielens 2001; Veen et al. 1998), and Marchenko et al. (2002) find a characteristic size of $0.5 \mu \mathrm{m}$ for the extended emission in WR 112. The model presented here also favors large grains with a quasi-uniform grain size ranging from 0.5 to $0.6 \mu \mathrm{m}$, with a standard interstellar distribution scaling as $a^{-3.5}$ (Mathis et al. 1977). A model with sizes ranging from 0.03 to $2.0 \mu \mathrm{m}$ also shows reasonable agreement with the data for similar values of the other parameters. Despite extensive trials, we were unable to find a distribution of smaller grain sizes that agreed with the data, in particular, the flux at the shorter infrared wavelengths. 


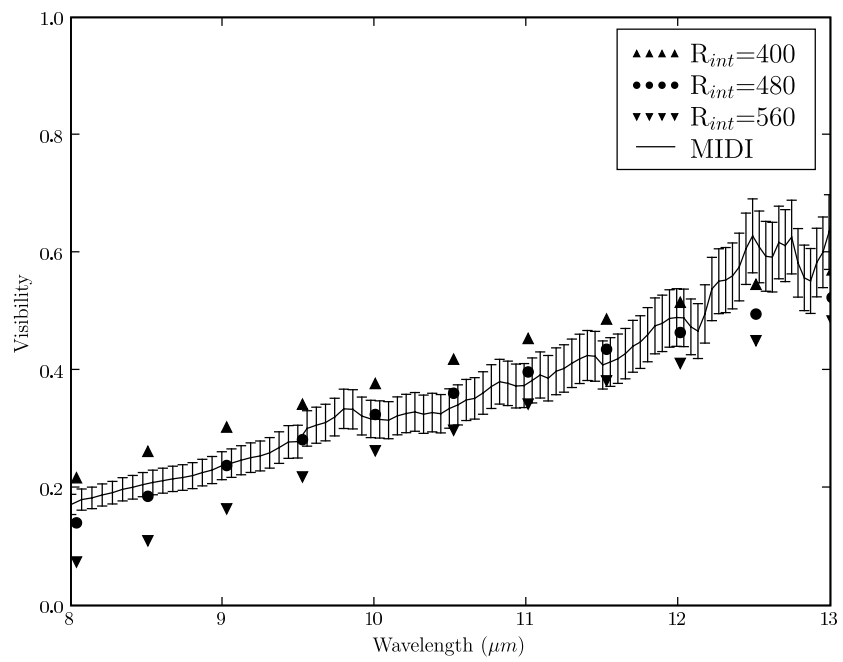

FIG. 11.-Visibility vs. wavelength for WR 95. The line (with error bars) shows the MIDI measurement; the filled circles represent the visibility points from the best model. For comparison, we show visibilities for models with different inner radii $\left(R_{\text {int }}\right)$ for the shell.

The optical depth for these dust shells in the mid-IR is fairly low (Zubko 1998). We use here a value of 0.015. Our primary goal of matching the measured visibilities is not strongly affected by the optical depth, and it is the spectra that constrain this parameter.

The important constraint imposed by the visibility profile is on the range of inner edge radii of the dust shell. The "best" model in Figure 7 has a value of $280 R_{*}$ for the inner edge radius, which is the same as in the WvdHT87 model. We show in Figure 8 the model visibility profile for values ranging from $230 R_{*}$ to $370 R_{*}$. Even for this narrow range, the visibility profiles are clearly different, indicating the fairly strong constraints imposed by the measurements.

The overdensity (denser than the $r^{-2}$ profile) that we introduce in the shell is to accommodate the visibilities measured with the smaller baselines at the Keck, which are lower than expected for a $r^{-2}$ profile consistent with the longer baseline MIDI visibilities. The overdense region in the dust shell extends from the inner edge $\left(R_{\text {int }}\right)$ up to $14 R_{\text {int }}$ in the model, i.e., size scales from 9 to 125 mas. This enhancement at the spatial scales probed by the segmenttilting experiment (resolution $\sim 50$ mas; see $\S 3.2$ ) decreases the visibility at the low spatial frequencies. This of course is not a unique solution, but offers an intuitive physical model. The overdensity could indicate an episode of more vigorous dust production. It could also be an artifact of applying an overly simplistic spherically symmetric model to a possibly complex shell geometry as in, for example, a pinwheel structure. In the absence of more complete visibility sampling, we limit ourselves to simply pointing out the existence of an extended structure in the dust around WR 106.

\subsection{WR 95}

WR 95 is also a late-type dust-forming WC, similar to WR 106. As in the case of WR 106, there is no definite evidence so far, either spectroscopic or photometric, of binarity. WR 95 falls below the sensitivity limit of the Keck segment-tilting experiment, and so we base our models solely on the MIDI visibilities and spectra. Our best model visibilities, along with the measured values in the $8-13 \mu \mathrm{m}$ MIDI band, are shown in Figure 11. Figure 12 shows the measured 8-13 $\mu \mathrm{m}$ SED, the near-IR and far-IR photometry from the literature, and the model SED profile. The

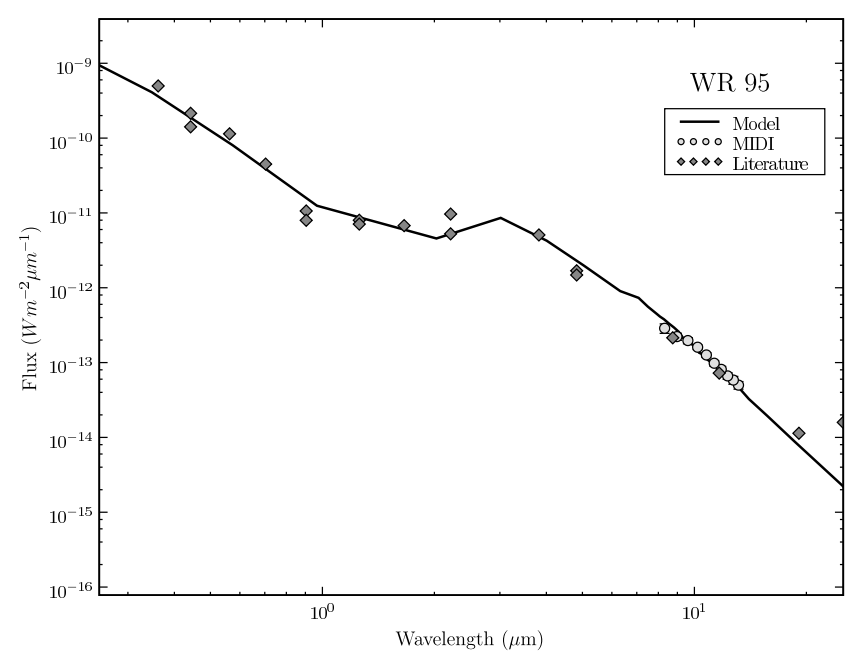

FIG. 12.-SED for WR 95. The solid line shows our best model, the gray circles indicate the measurements from MIDI (dereddened as in text), and the diamonds indicate measurements from the literature (see text for sources)

dust temperature profile is as in Figure 13. The parameters of the model are as below.

\subsubsection{Central Star}

1. Effective temperature $T_{\text {eff }}=25,000 \mathrm{~K}$.

2. Angular radius of $\operatorname{star} R_{*}=3.2 \times 10^{-5}$ arcsec.

\subsubsection{Dust Shell Physical Properties}

1. The composition is amorphous carbon.

2. The grain size ranges from 0.5 to $0.6 \mu \mathrm{m}$.

3. The optical depth $\tau=0.005$ at $10 \mu \mathrm{m}$.

\subsubsection{Dust Shell Geometry}

1. The inner radius of the dust shell $R_{\text {int }}=480 R_{*}$ (multiples of stellar radius).

2. The outer radius of the dust shell is $1230 R_{*}$.

3. The density profile is proportional to $r^{-2}$.

As in the case of WR 106, we base our initial model parameters mostly on WvdHT87. To match the near IR photometry and our visibility measurements, we had to adopt higher temperature of

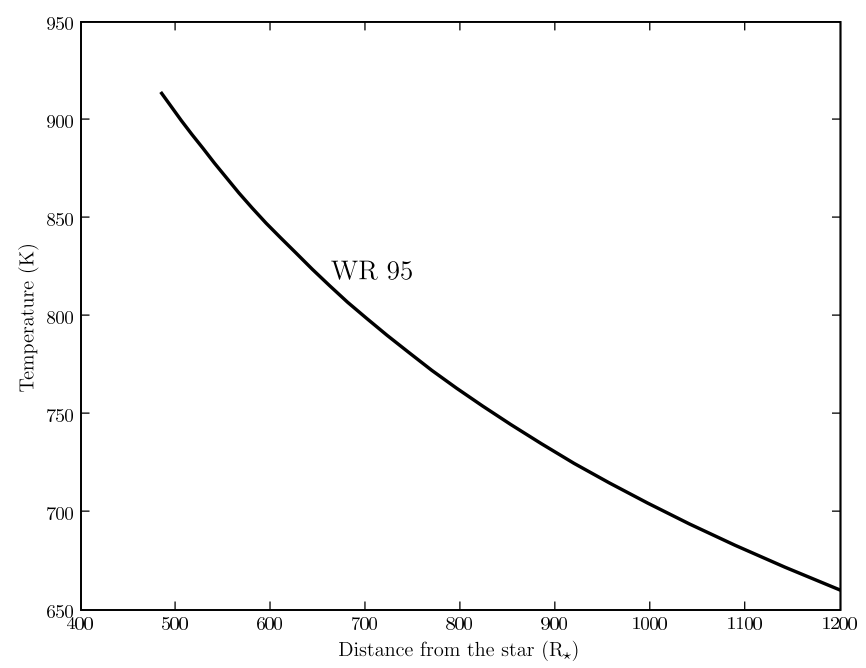

FIG. 13.-Dust temperature profile of our WR 95 model. Distance from the star is in units of $R_{*}$. 
25,000 K for the WR star. The optical depth for our best model, 0.005 at $10 \mu \mathrm{m}$, is lower than that for WR 106. Since we do not have shorter baseline Keck observations in this case to detect extended structure, a uniform $r^{-2}$ density profile suffices. The nominal inner radius of the shell is $480 R_{*}$ (Fig. 11 shows how the model visibilities vary for slightly different values of this parameter), compared to $410 R_{*}$ derived by WvdHT87. As in the case of WR 106, a dust grain distribution with sizes ranging from 0.3 to $2.0 \mu \mathrm{m}$ also yields a fair fit to the measurements.

\section{CONCLUSIONS}

High-resolution measurements of dust around massive stars are sparse in the literature. Coupled with the rarity of the objects themselves, it is not surprising that so much remains unclear about the mechanisms of dust formation and the nature of the dust itself. Only a handful of WR stars known to produce dust have been imaged with any degree of detail. Three of these, WR 104, WR 98a, and WR 140 are binaries, producing dust at the windwind interface. The fourth, WR 112, reveals a multiple-shell dust structure, although at much larger (arcsecond) scales than discussed here for our targets, also suggestive of a binary (Marchenko et al. 2002). Hence, the measurements presented here, albeit with limited baselines, of similar objects with no previous evidence of binarity, are an important step in probing the nature of these dust shells.

We observed two WC9 stars (WR 106 and WR 95), an LBV (AG Car), and an object that may be in a stage of transition from an LBV to a WR phase, NaSt 1. NaSt 1 was well resolved, and the visibility measurements suggest increasing size with wavelength, a behavior unlike the WC 9 stars in our sample. We favor a two-component model for the dust with a compact, unresolved core and an extended component. Previous studies of the SED of this object have also indicated this structure. For AG Car as well, our measurements resolve a compact mid-IR source that is likely associated with a known molecular gas component and is the first direct evidence for dust near the central star. For both these objects, this study has revealed the presence of close-in dust that has important implications for the state of evolution of the central star.

For the WC stars, we construct spherically symmetric radiative transfer models for the dust shells. These models indicate that the inner edge of these shells are at a few hundred stellar radii (tens of AU) from the star. Gaussian sizes (FWHM) directly estimated from the visibility measurement provide model-independent upper limits that are consistent with these values. At these distances, the dust temperature is fairly close to sublimation values for carbonaceous dust. In addition, our results support previous indications of fairly large grains $(\sim 1 \mu \mathrm{m})$, despite these conditions. These new results reopen an existing controversy: How does dust form in the hostile environment of a WR stellar wind at such high temperatures? The proximity of the inner edge to the star also forces us to adopt values close to $20,000 \mathrm{~K}$ for the central star to keep the dust below the sublimation point. However, the spectra of late-type WC stars point to higher $(40,000$ to $70,000 \mathrm{~K})$ temperatures, rendering the situation even worse. Our results certainly indicate that dust formation is likely occurring under fairly extreme conditions. This may suggest a colliding-wind binary model, but direct evidence is still lacking for the objects in our sample.

The dust around WR 106 seems to deviate from an $r^{-2}$ profile at the shorter baseline Keck measurements. Our simple models suggest an overdense region fairly close to the inner edge. This could also be a first indication of binarity, with multiple shells from episodic dust production or a more complex geometry, as in a pinwheel. But given our limited baseline coverage, a caveat on modeling artifacts is warranted.

We have not attempted to estimate the dust mass from the models. Our measurements are insensitive to the colder dust farther from the star, and it is unlikely that we can improve on the existing dust mass estimates for these stars from spectral profiles.

In the long term, further progress would involve multiple baselines to image the dust structure. Observations of the outer dust at longer wavelengths are also required to accurately estimate the dust mass and geometry. In the short term, we aim to look for asymmetries in the dust distribution by using the UT3-UT4 baseline at the VLTI, orthogonal to the one used here.

This work was performed in part under contract with the Jet Propulsion Laboratory (JPL) funded by NASA through the Michelson Fellowship Program. JPL is managed for the National Aeronautics and Space Administration (NASA) by the California Institute of Technology. Some of the data presented herein were obtained at the W. M. Keck Observatory, which is operated as a scientific partnership among the California Institute of Technology, the University of California, and NASA. The observatory was made possible by the generous financial support of the W. M. Keck Foundation. The authors wish to recognize and acknowledge the very significant cultural role and reverence that the summit of Mauna Kea has always had within the indigenous Hawaiian community. We are most fortunate to have the opportunity to conduct observations from this mountain. We acknowledge the VLTI consortium for both technical and software support.

We thank the referee for comments that have significantly improved this article.

Facilities: Keck:I, VLTI
Barniske, A., Oskinova, L., Hamann, W.-R., \& Gräfener, G. 2006, in ASP Conf. Ser. 353, Stellar Evolution at Low Metallicity: Mass Loss, Explosions, Cosmology (San Francisco: ASP), 241

Cherchneff, I., Le Teuff, Y. H., Williams, P. M., \& Tielens, A. G. G. M. 2000, A\&A, 357, 572

Chiar, J. E., \& Tielens, A. G. G. M. 2001, ApJ, 550, L207

Clark, J. S., Egan, M. P., Crowther, P. A., Mizuno, D. R., Larionov, V. M., \& Arkharov, A. 2003, A\&A, 412, 185

Coude Du Foresto, V., Ridgway, S., \& Mariotti, J.-M. 1997, A\&AS, 121, 379

Crowther, P. A. 2003, Ap\&SS, 285, 677

Crowther, P. A., \& Smith, L. J. 1999, MNRAS, 308, 82

Danchi, W. C., Bester, M., Degiacomi, C. G., Greenhill, L. J., \& Townes, C. H. 1994, AJ, 107, 1469

Groh, J. H., Hillier, D. J., \& Damineli, A. 2006, ApJ, 638, L33

Harries, T. J., Monnier, J. D., Symington, N. H., \& Kurosawa, R. 2004, MNRAS, 350, 565
Humphreys, R. M., \& Davidson, K. 1979, ApJ, 232, 409 1994, PASP, 106, 1025

Humphreys, R. M., Lamers, H. J. G. L. M., Hoekzema, N., \& Cassatella, A. 1989, A\&A, 218, L17

Lamers, H. J. G. L. M., Nota, A., Panagia, N., Smith, L. J., \& Langer, N. 2001, ApJ, 551, 764

Leinert, C., et al. 2003, Proc. SPIE, 4838, 893

. 2004, A\&A, 423, 537

Marchenko, S. V., Moffat, A. F. J., Vacca, W. D., Côté, S., \& Doyon, R. 2002, ApJ, 565, L59

Massey, P., Plez, B., Levesque, E. M., Olsen, K. A. G., Clayton, G. C., \& Josselin, E. 2005, ApJ, 634, 1286

Mathis, J. S., Rumpl, W., \& Nordsieck, K. H. 1977, ApJ, 217, 425

Mermilliod, J.-C., \& Mermilliod, M. 1994, Catalogue of Mean UBV Data on

Stars VI (Berlin: Springer)

Monet, D. G., et al. 2003, AJ, 125, 984 
Monnier, J. D., Tuthill, P. G., \& Danchi, W. C. 1999, ApJ, 525, L97

Monnier, J. D., Tuthill, P. G., Danchi, W. C., Murphy, N., \& Harries, T. J. 2007, ApJ, 655, 1033

Monnier, J. D., Tuthill, P. G., Ireland, M. J., Cohen, R., \& Tannirkulam, A. 2004a, BAAS, 36, 1367

Monnier, J. D., et al. 2004b, ApJ, 602, L57

Niccolini, G., Woitke, P., \& Lopez, B. 2003, A\&A, 399, 703

Nota, A., Leitherer, C., Clampin, M., Greenfield, P., \& Golimowski, D. A. 1992, ApJ, 398, 621

Nota, A., Pasquali, A., Marston, A. P., Lamers, H. J. G. L. M., Clampin, M., \& Schulte-Ladbeck, R. E. 2002, AJ, 124, 2920

Ochsenbein, F., Bauer, P., \& Marcout, J. 2000, A\&AS, 143, 23

Pascucci, I., Wolf, S., Steinacker, J., Dullemond, C. P., Henning, T., Niccolini, G., Woitke, P., \& Lopez, B. 2004, A\&A, 417, 793

Rouleau, F., \& Martin, P. G. 1991, ApJ, 377, 526

Sloan, G. C., Kraemer, K. E., Price, S. D., \& Shipman, R. F. 2003, ApJS, 147, 379

Smith, J. D. T., \& Houck, J. R. 2001, AJ, 121, 2115

Smith, L. F. 1968, MNRAS, 138, 109

Smith, L. J., Nota, A., Pasquali, A., Leitherer, C., Clampin, M., \& Crowther, P. A. 1998, ApJ, 503, 278

Turon, C., et al. 1993, Bull. Inf. CDS, 43, 5

Tuthill, P. G., Monnier, J. D., \& Danchi, W. C. 1999, Nature, 398, 487

Tuthill, P. G., Monnier, J. D., Danchi, W. C., Wishnow, E. H., \& Haniff, C. A. 2000, PASP, 112, 555

Tuthill, P., Monnier, J., Tanner, A., Figer, D., Ghez, A., \& Danchi, W. 2006, Science, 313, 935
Usov, V. V. 1991, MNRAS, 252, 49

van der Hucht, K. A. 2001, NewA Rev., 45, 135

van der Hucht, K. A., Conti, P. S., Lundstrom, I., \& Stenholm, B. 1981, Space Sci. Rev., 28, 227

van der Hucht, K. A., Williams, P. M., \& Morris, P. W. 2001, in The Promise of the Herschel Space Observatory, ed. G. L. Pilbratt, J. Cernicharo, A. M. Heras, T. Prusti, \& R. Harris (SP-460; Noordwijk: ESA), 273

Veen, P. M., van Genderen, A. M., van der Hucht, K. A., Li, A., Sterken, C., \& Dominik, C. 1998, A\&A, 329, 199

Voors, R. H. M., Waters, L. B. F. M., Morris, P. W., Trams, N. R., de Koter, A., \& Bouwman, J. 1999, A\&A, 341, L67

Voors, R. H. M., et al. 2000, A\&A, 356, 501

Weiner, J., et al. 2006, ApJ, 636, 1067

Williams, P. M. 1995, in IAU Symp. 163, Wolf-Rayet Stars: Binaries; Colliding Winds; Evolution, ed. K. A. van der Hucht \& P. M. Williams (Dordrecht: Kluwer), 335

Williams, P. M., \& van der Hucht, K. A. 2000, MNRAS, 314, 23

Williams, P. M., van der Hucht, K. A., Pollock, A. M. T., Florkowski, D. R., van der Woerd, H., \& Wamsteker, W. M. 1990, MNRAS, 243, 662

Williams, P. M., van der Hucht, K. A., \& The, P. S. 1987, A\&A, 182, 91 (WvdHT87)

Williams, P. M., et al. 2001, MNRAS, 324, 156

Woitke, P., \& Niccolini, G. 2005, A\&A, 433, 1101

Zubko, V. G. 1998, MNRAS, 295, 109 